\title{
Influence de la période de la houle sur la stabilité des digues à talus
}

\author{
Influence of wave period \\ on the stability of breakwaters
}

L. Carpentier

F. Brosselard-Faidherbe

Laboratoire central d'hydraulique de France

Maisons-Alfort

Les formules usuelles de stabilité des digues ignorent, entre autres, l'influence de la période de la houle sur la tenue des carapaces, alors que le coefficient de stabilité de Hudson peut varier dans le rapport de 1 à 3 , en raison de cette influence.

Les études expérimentales décrites dans l'article ont confirmées le rôle de la période du point de vue qualitatif, à défaut d'avoir permis d'exprimer les lois générales correspondantes, en l'état actuel des recherches.

The usual stability formula of dams do not consider the effect of wave period on the stability of breakwater revetments, whereas the stability coefficient of Hudson can vary within the ratio of 1 to 3 because of this effect.

The experimental studies described in the article have confirmed the role of the period from the qualitative point of view, but have not enabled the general corresponding laws to be expressed, in the current state of research.

L'influence de la période de la houle sur la stabilité des digues à talus est complexe et importante. Aussi, d'aucuns se sont-ils préoccupés d'introduire ce paramètre dans la formule de stabilité la plus usuelle, celle de Hudson, laquelle a le mérite d'être simple et utile même avec des éléments autres que les blocs naturels pour lesquels elle a été conçue. C'est dans cet esprit que le Service technique central (STC) de la Division des ports maritimes a confié au Laboratoire central d'hydraulique de France (LCHF), familier depuis longtemps déjà de cette notion de com- plexité de la période, un programme d'études expérimentales dans un cas volontairement simplifié à l'extrême, celui d'un talus raide recouvert de cubes rainurés.

Inventés par le Port autonome du Havre avec la collaboration du LCHF, les cubes rainurés sont en effet l'optimum technico-économique incontesté des éléments artificiels massifs et robustes qui autorisent, de par leur comportement évolutif, à ne pas surinvestir pour pallier les agressions de quelques vagues pouvant se présenter seulement tous les 10 ou 20 ans. 


\section{Réflexions préliminaires sur l'orientation des recherches}

Les paramètres parmi les plus significatifs intervenant dans la détermination de la stabilité d'une digue sont au nombre d'une quarantaine au moins; ils appartiennent à trois domaines: l'eau (en particulier les caractéristiques des agitations, hauteur, période, direction, spectre d'énergie, durée, groupement de vagues, forme des vagues, etc.), les fonds (notamment, profondeur, pente, nature, rugosité, porosité, etc.) et l'ouvrage (pente de talus, cote du couronnement, dimension, densité, arrangement et compacité des blocs de carapace, caractéristiques des sous-couches et du noyau, etc.), qui interfèrent de façon complexe.

La vingtaine de formules de stabilité habituellement répertoriées ne fait intervenir qu'un petit nombre de paramètres caractérisant les seuls domaines eau et ouvrages, et on peut s'étonner de l'absence du paramètre fonds qui est pourtant un des éléments conditionnant « l'agressivité ") des vagues.

Parmi ces formules, seulement une dizaine prend en compte l'influence de la période, et encore, de façon trop artificielle, ou tout simplement erronée.

La formule de Hudson, la plus usitée actuellement parce que plus simple et réaliste, est aussi celle qui tient le mieux compte de la période puisqu'elle fait intervenir des coefficients différents suivant que la houle déferle ou non.

C'est donc tout naturellement que depuis plusieurs années, les dommages subis dans les digues en eau profonde ont motivé, autour de plusieurs thèmes, des recherches dans le cadre de l'application de la formule de Hudson.

L'influence de la période est l'un de ces thèmes, et la préoccupation qui nous a guidés a été de rechercher la ou les périodes critiques d'agressivité ainsi que le domaine dangereux du couple hauteur-période situé entre les clapots de vents locaux (ondes courtes) et les ondes de longue période comme les seiches.

Le programme d'expérimentation a consisté d'une part en une étude du comportement des éléments de carapace (stabilité globale), d'autre part en une analyse des efforts hydrodynamiques sur quelques éléments et des mouvements de l'eau dans la carapace, ceci avec le même ouvrage représenté à l'échelle géométrique du 1/50 (fonds horizontaux à la cote $-32,5 \mathrm{~m}$; talus à $4 / 3$; cubes de $6 \mathrm{~m}^{3}$ ) et soumis à des houles de 8 à $25 \mathrm{~s}$ de période.

\section{Etudes expérimentale de la stabilité globale}

\subsection{Résultats de l'expérimentation}

L'expérimentation a été conduite avec des vagues régulières de période constante dont la hauteur était augmentée par paliers successifs de durée correspondant à $6 \mathrm{~h} /$ nature jusqu'à obtenir si possible la ruine de l'ouvrage.

La série d'essais a montré que la plage de période où l'agressivité des vagues est grande est étendue, et que cette agressivité dépend du niveau de dommages considéré.
En première approximation, il existe entre les dommages cumulés $D$ et les autres paramètres, une relation du type $D($ en $\%)=A(T)(K-K o)$

avec $A$ : coefficient dépendant de la période $T$,

$K$ : coefficient de stabilité de Hudson,

$K o$ : coefficient de Hudson pour apparition des premiers dommages.

Le tableau ci-après donne les valeurs de $A$ obtenues dans les conditions d'essai :

\begin{tabular}{|l|c|c|c|c|c|c|c|}
\hline$T$ en $s$ & 8 & 10 & 12 & 14 & 16 & 20 & 25 \\
$A$ & 0,56 & 1,07 & 1,01 & 0,93 & 0,86 & 0,63 & 0,80 \\
\hline
\end{tabular}

La formule de Hudson pourrait alors s'écrire :

$$
P \sim \frac{\rho g H^{3}}{K o+D / A}
$$

dans laquelle :

$P$ est le poids des blocs,

$\rho g$ est le poids spécifique des blocs,

$H$ est la hauteur de la houle,

- avec pour $A$ soit sa valeur maximale caractérisant avec $K o$ le type de bloc utilisé, soit une valeur pondérée en fonction de la période et de la taille des blocs.

Aux périodes de 10 à $12 \mathrm{~s}$ sont apparus les déferlements par " effondrement " qui ont conduit le plus rapidement à la ruine par perte d'unités ou de groupes de cubes au droit du point d'impact des lames.

Avec la période de $20 \mathrm{~s}$, la ruine est intervenue par fluidisation de la carapace, sans relation avec les déferlements : suite à un champ de fortes vitesses qui a pénétré profondément dans l'ouvrage, des blocs ont été soulevés simultanément tant à la montée qu'à la descente de la lame.

\subsection{Conclusions}

Outre la mise en évidence d'un phénomène particulier pour la période de $20 \mathrm{~s}$, les recherches bibliographiques et les essais de stabilité globale nous ont conduit aux convictions suivantes :

a) Pour la gamme des périodes agressives, le coefficient de stabilité $K$ de Hudson varie de 1 à 3 . Si $T m$ est la période minimale produisant des oscillations ou des déplacements de cubes quand le déferlement par glissement est atteint, les périodes classiquement les plus défavorables valent 1,3 à $1,5 \mathrm{Tm}$, périodes que l'on rencontre bien en nature. La ruine de l'ouvrage peut se produire pour des périodes exceptionnelles (2,5 $\mathrm{Tm}$ dans les conditions d'essai) par fluidisation de la carapace. Au-delà, les vagues perdent leur agressivité.

b) La période intervient par son rôle dans la détermination des types de déferlement; ce sont ceux par " effondrement " - les plus concentrés et les moins émulsionnés - qui sont les plus agressifs (10 et $12 \mathrm{~s}$ ici). Elle intervient aussi par un effet de masse non émulsionnée (ici $20 \mathrm{~s})$.

c) Le nombre d'Iribarren ne peut à lui seul décrire les déferlements, même pour les ouvrages «en eau profonde ". En effet ce qualificatif est utilisé dès que ia 


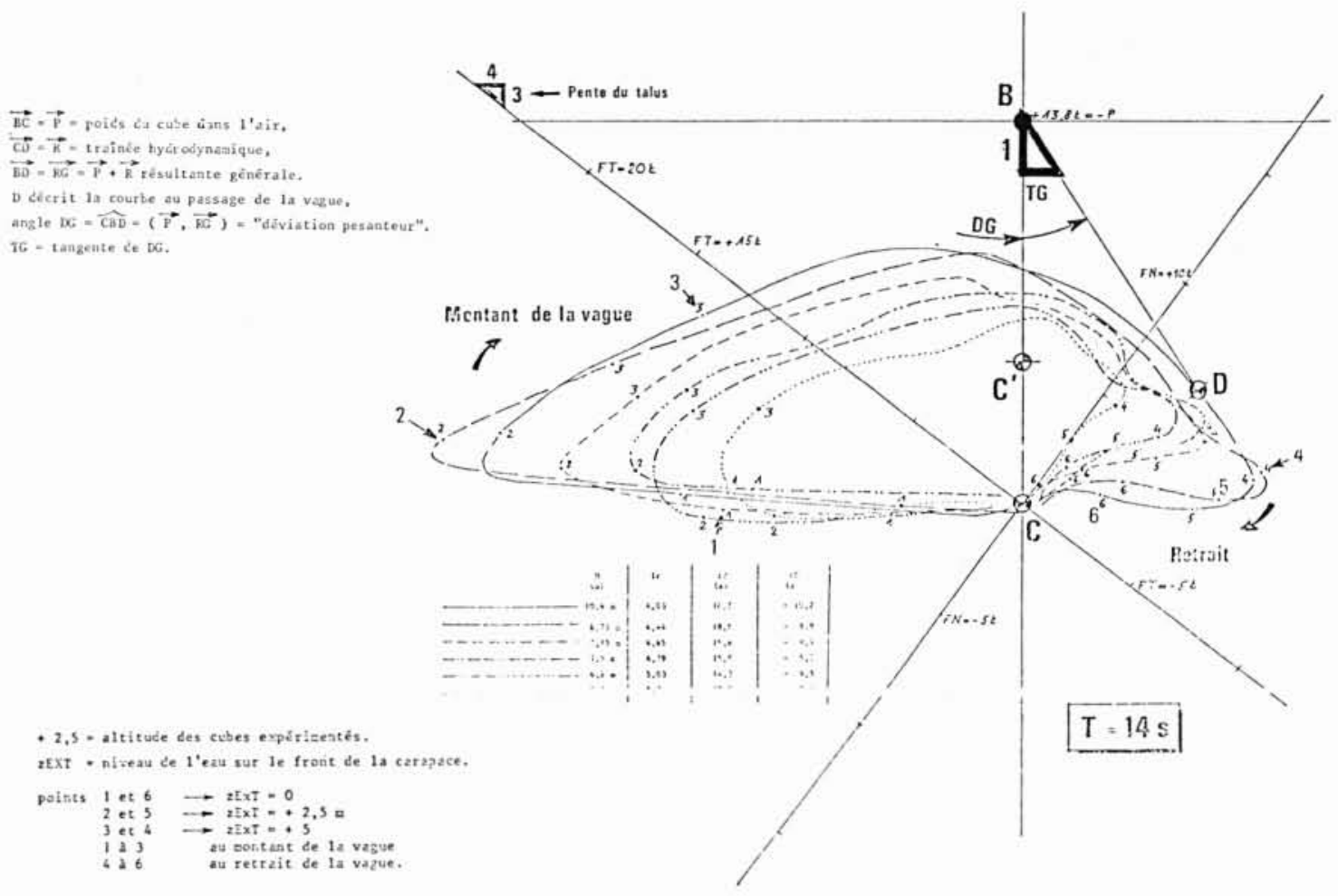

profondeur $d$ est supérieure à $3 \mathrm{H}$, et comme les vagues "sentent" le fond dès que le rapport de la longueur d'onde $L$ à la profondeur $d$ dépasse 2, c'est une condition facile à atteindre. Pratiquement, on peut affirmer que le déferlement dépend aussi des fonds dès qu'il passe le stade de la volute*.

d) Dans une houle naturelle, la dispersion des caractéristiques des lames favorise les déferlements sur les digues; l'attaque de la houle gagne en importance quand croissent profondeur et pente des fonds. Ces deux paramètres ont autant, sinon plus, d'influence que la période sur la stabilité d'un ouvrage : ils conduisent à faire croitre la hauteur de la vague de calcul car ils limitent les hauteurs des lames susceptibles d'atteindre la digue et contribuent à en conditionner l'agressivité.

e) L'avènement de vagues exceptionnelles est d'autant plus probable que la tempête est longue; en outre, comme la croissance des dégâts est souvent géométrique, la durée des tempêtes est un paramètre important dans la détermination de la pérennité d'une digue; sa prise en compte conduit tout naturellement à celle de "pourcentage de dommages " qui constitue un paramètre difficile à appréhender.

* Rappelons que le nombre d'Iribarren $I r=\operatorname{tg} \alpha / h / L_{m}$ ou $\alpha$ est l'angle du talus, $h$ la hauteur des vagues et $L_{v}$ la longueur d'onde au large, est généralement utilisé pour caractériser les différents types de déferlement.

\section{Mesures de la trainée et du niveau d'eau dans la carapace}

Après avoir reconstruit et rigidifié la carapace des essais précédents, deux types d'analyse avec transfert et traitement en temps réel des informations sur ordinateur ont été entrepris :

- 4 cubes de la couche externe situés à la cote $z E X T=+2,5 \mathrm{~m}$ au-dessus du niveau de repos ont été isolés légèrement de leurs voisins et tenus par une balance à 6 composantes de mesure des efforts globaux;

- 2 sondes ont été placées pour suivre les fluctuations $z E X T$ et $z I N T$ du niveau de l'eau à la surface de la carapace et sur son interface avec la sous-couche.

Dans la mesure du possible, on a tenté de refaire les mêmes essais qu'avec la carapace destructible, mais parfois des déferlements différents furent observés du fait des modifications des caractéristiques - pente et porosité - subies par la carapace au cours des essais antérieurs, suite à son tassement sous l'action des fortes houles.

\subsection{Analyse de la traînée et recherche d'un critère d'instabilité (TG)}

Pour procéder à cette analyse, la variation dans le temps du vecteur traînée $C D$ mesuré par la balance a été 
examinée, et une représentation sous forme de rose en a été faite.

Un exemple est donné sur la figure de la page précédente pour $T=14 \mathrm{~s}$ et des hauteurs $H$ de la houle incidente allant de 5 à $10 \mathrm{~m}$ :

$B C$ et $B C$ 'étant les vecteurs poids d'un cube $(13,8 \mathrm{t}$ dans l'air ou 7,92t dans l'eau), $B D$ est la résultante générale des forces extérieures agissant sur le cube.

$A$ la montée de la vague, $D$ décrit rapidement le lobe gauche de la rose : la traînée $C D$ est forte, horizontale et brève, et la résultante $B D$ est perpendiculaire au talus qui se tasse, se compacte et se consolide. Avec des périodes plus longues, la pointe s'arrondit : toute une zone de la carapace est comprimée (traînée horizontale égale au poids pour $T=25 \mathrm{~s}$ et $H=10 \mathrm{~m}$ ). Avec des périodes plus courtes, la pointe s'accentue : la traînée devient un effort violent et bref (choc) qui enchâsse à cette altitude les cubes les uns dans les autres (une et deux fois le poids pour $H=5$ et $7.5 \mathrm{~m}$ avec $T=10 \mathrm{~s})$. A cette cote, les risques de dégradations sont nuls au montant puisqu'il y a consolidation de la carapace, mais de façon très différente selon la période.

$A u$ retrait de la vague, le lobe de droite est décrit lentement par $D$, et la résultante $B D$ garde toujours un module voisin du poids. Elle fait avec la verticale un angle $D G$ qui est donc une déviation apparente de la pesanteur. Le retrait de la vague équivaut alors à un raidissement du talus pendant une fraction notable de la période; ainsi le maximum de la déviation DGmax (ou sa tangente $T G \max =\operatorname{tg} D G \max )$ peut être considéré comme un indice d'instabilité qu'il est intéressant d'analyser :

- pour $T G=14 \%$ ou $29 \%$ seulement, le talus réel à $4 / 3$ "passe " en apparence à $1 / 1$ ou $3 / 4$, c'est-à-dire qu'il s'affaisserait si sa construction était tentée dans de semblables conditions;

- pour $T=14 \mathrm{~s}$ et $H$ supérieur à $8 \mathrm{~m}, T G \max$ atteignait $68 \%$ environ, ce qui signifie que le talus passe de $4 / 3$ à presque 1/3. Lors des essais de stabilité globale, ce domaine de houles a été atteint sans ruine mais avec des dommages (15 à $20 \%$ ); il est à supposer que des tassements, compactages et déformations de la carapace ont certainement entraîné une modification sensible de la traînée à prendre en considération.

Pour les cubes étudiés, c'est-à-dire situés peu au-dessus du niveau de l'eau dans une carapace figée dans son état initial, on a trouvé que le paramètre d'instabilité $T G$ variait comme le carré de la hauteur des vagues. Ainsi, dans le système $M T S$ :

- pour $T=10 \dot{a} 16 \mathrm{~s}$ environ $T G=(0,11 \mathrm{H})^{2}$ plafonnant vers 0,6 à 0,7 ;

- pour $T=20 \mathrm{~s}$ sensiblement plus avec $T G=(0,135 \mathrm{H})^{2}$ plafonnant vers 0,8 ;

- pour $T=8 \mathrm{~s}$ ou $25 \mathrm{~s}$ beaucoup moins avec $T G=(0,07 H)^{2}$.

\subsection{Analyse de la montée des vagues sur et dans la carapace}

L'enregistrement et l'analyse informatique des fluctuations de la surface libre ont donné les résultats intéressants suivants : a) excepté pour les fortes houles qui conduisent à des valeurs moindres, on a trouvé que le rapport de la montée de la vague zu sur la carapace (run up) à la hauteur de la houle incidente $H$ :

- valait environ 2 pour $T=10$ à $16 \mathrm{~s}$,

- était maximum avec la valeur 2,6 pour $T=20 \mathrm{~s}$.

- diminuait pour $T=8$ et $25 \mathrm{~s}$ (respectivement 1,2 et 1,5 ).

b) Pour des hauteurs de houle modérées, le découvert de la carapace $z D$ atteint un plafond qui croit avec la période : $z D \max =4,5 \mathrm{~m}$ avec $8 \mathrm{~s}, 7 \mathrm{~m}$ avec $10 \mathrm{~s}$ et 9,5 à $10 \mathrm{~m}$ avec $14,16,20$ et $25 \mathrm{~s}$.

c) Le rapport de la dénivelée totale sur la carapace $\mathrm{He}$ à la hauteur incidente $(\mathrm{He}: H)$ croît rapidement puis lentement de $8 \mathrm{~s}$ à $20 \mathrm{~s}$ et chute fortement entre 20 et $25 \mathrm{~s}$ :

- $H e=2+1,1 \mathrm{H}$ avec $T=8 \mathrm{~s}$

- $H e=4 H$ pour $H=3,5 m$ à $6,5 \mathrm{~m}$

ou $20+H$ pour $H=6.5 m$ à $10 \mathrm{~m}$ avec $T=20 \mathrm{~s}$

- $2 \mathrm{H}$ pour $T=25 \mathrm{~s}$

d) La transmission de l'onde à travers la carapace s'amplifie avec la période mais de manière non uniforme. Les rapports de la montée $z u^{\prime}$ et de la dénivelée totale sous la carapace Hint à la montée sur la carapace $\left(z u^{\prime}: z u\right.$ et Hint : $z u$ ) varient linéairement avec la période suivant les expressions suivantes (en MTS) :

- $z u^{\prime} z u=0,075(T-3)$ pour $T=8 \dot{a} 14 \mathrm{~s}$ $=0,75+0,025 T$ pour $T=14 \dot{a} 25 s$.

$$
\frac{20 \mathrm{Hint}}{T(z u+T)}=\begin{aligned}
& 1 \quad \text { pour } T=8 \text { à } 16 \mathrm{~s} \\
& 0,87 \text { pour } T=20 \mathrm{~s} \\
& 0,72 \text { pour } T=25 \mathrm{~s}
\end{aligned}
$$

e) Le déphasage en temps du passage de la surface libre au niveau $+2,5 \mathrm{~m}$ dépend assez peu de la montée $z u$ sur la carapace et de la période pour $T=10$ à $25 \mathrm{~s}$ :

- à la montée, il décroît avec $z u$ et croît avec $T$,

- à la descente, il croît avec $z u$ et tend à décroître avec $T$.

f) Curieusement, à la montée, le déphasage en altitude ne dépend pas du run up et peu de la période, mais beaucoup de l'altitude sur la carapace. La pente moyenne du fond de l'onde dans l'épaisseur de la carapace qui traduit ce déphasage vaut :

- quand le niveau de repos est atteint sur l'extérieur de la carapace : de $T=8$ à $20 \mathrm{~s}$ et tombe de $31,3^{\circ}$ à $22,4^{\circ}$ entre 20 et 25 ,

- quand $+2,5 \mathrm{~m}$ est atteint pf $2=40^{\circ}+\mathrm{T} .1,5^{\circ}$ de $T=8$ à $16 \mathrm{~s}$ ou $94^{\circ}$ et $69^{\circ}$ pour 20 et $25 \mathrm{~s}$, c'est-à-dire que pour $20 \mathrm{~s}$ le front de l'onde a dépassé la verticale.

g) Au retrait le déphasage en altitude est fonction de $z u$ mais peu de la période; quand la cote $+2,5 \mathrm{~m}$ découvre sur la carapace, la base de celle-ci est encore mouillée sur une hauteur qui est en moyenne plus haute d'environ :

- $0,45(z u-5 \mathrm{~m})$ jusqu'à $z u=15 \mathrm{~m}$, puis

- $0,75(z u-9 \mathrm{~m})$ pour $z u=15$ à $20 \mathrm{~m}$.

h) Sur $5 \mathrm{~m}$ au-dessus du niveau de repos, la célérité de la surface libre sur la carapace dépend peu de la période entre 8 et $20 \mathrm{~s}$ et conduit (en MTS) à :

- $V / H=0,7$ à 1,1 à la montée et

de l'ordre de 0,65 au retrait,

- mais $V / H=0,33$ à la montée et 0,38 au retrait pour $T=25 \mathrm{~s}$. 


\subsection{Conclusions}

Pour des cubes rainurés situés peu au-dessus du niveau de repos, l'étude de la traînée a porté un nouvel éclairage sur le processus des déformations/tassements bien connu des expérimentateurs : il se produit à la montée des lames, même de courtes périodes.

Dans la zone située juste au-dessus du niveau de repos de l'eau, l'instabilité résulte du retrait de la lame qui produit l'équivalent d'une déviation apparente de la pesanteur dont la tangente $T G$ a fait l'objet de notre analyse.

La stabilité globale comme l'indice d'instabilité $T G$ et certaines caractéristiques de l'onde sur ou dans la carapace, suivent une certaine loi entre 8 et $16 \mathrm{~s}$. Mais on trouve pour $20 \mathrm{~s}$ des contraintes supérieures et avec $25 \mathrm{~s}$ très inférieures à celles de cette loi. L'existence d'une période critique a donc été mise en évidence. Il apparaît souhaitable de poursuivre les recherches pour préciser la valeur de cette période critique et sa dépendance vis-à-vis d'un certain nombre de paramètres, notamment de la position des blocs dans la carapace.

L'étude qui a été faite montre l'intérêt de telles recherches avec de nouveaux types de mesures, mais plutôt que d'examiner finement l'influence de la période avec des vagues de hauteur uniforme - donc inconnues en nature - , il semble que l'on devrait s'attacher à la recherche des contraintes maximales produites par des houles irrégulières, ce qui devrait conduire à des formules plus directement utilisables par les ingénieurs.

Etant donné l'utilité de bien comprendre le mode d'action des houles pour établir ces formules, il y aura lieu, au cours des expérimentations, de procéder simultanément à l'analyse de la trainée d'éléments situés à différentes altitudes sur la carapace et au suivi du front de l'onde à travers la carapace.

M. BROSSELARD-FAIDHERBE : Avant de présenter sa communication, M. Brosselard-Faidherbe avait déclaré en réponse à l'exergue du Président Banal : effectivement on s'occupe encore des digues à talus. Avec des moyens d'étude plus puissants, des réalisations par plus grandes profondeurs et des désastres comme celui de Sinès, on se pose même de plus en plus de questions. Mais certaines sont résolues comme l'influence pénalisante pour les carapaces poreuses de l'obliquité de la houle. En pratique, la formule très simple de Hudson reste seule utilisée, mais avec un éventail de coefficients qui s'étend pour cerner les particularités et une permanente remise en question des valeurs à adopter dans les cas les plus classiques.

Outre la stabilité hydraulique de la carapace, on se préoccupe également aujourd'hui de la rupture des blocs et des risques de glissements d'ensemble, comme vient justement de la souligner le rapporteur général $\mathrm{M}$. Monadier. Il n'y a plus d'esprits attardés sur la conservation de la période ou la régularité des houles, mais pour les houles dites aléatoires à reproduire, on se demande quel spectre choisir et quels groupements de vagues. Pour cerner l'influence encore incertaine de ces paramètres, des comparaisons seraient souhaitables avec des mesures des efforts sur chaque bloc telles que celles que nous présentons, mais l'attention devrait aussi porter sur les effets et conditions de mise en émulsion des lames dans la carapace. Ce phénomène est en effet susceptible d'expliquer la chute d'agressivité entre les périodes critiques de $10 \mathrm{~s}$ et de $20 \mathrm{~s}$ observées dans les conditions de certaines de nos recherches, ainsi que certaines performances des ouvrages Jarlan ou Staemphli. Avant de présenter celles-ci, nous pouvons préciser que la chute d'agressivité constatée pour $25 \mathrm{~s}$ résulte - comme expliqué en 1966 au CIN de Stockholm par M. Iribarren - de ce que les circulations dans la carapace sont alors freinées par la lenteur de l'onde devant l'ouvrage. 